\title{
INFRARED CORONAL EMISSION LINES AND THE \\ POSSIBILITY OF THEIR LASER EMISSION IN SEYFERT \\ NUCLEI
}

\author{
MATTHEW A. GREENHOUSE \\ Laboratory for Astrophysics, National Air and Space Museum, Smithsonian Institution, \\ Washington, DC 20560 \\ URI FELDMAN \\ Solar Terrestrial Relationships Branch, Naval Research Laboratory, Washington, DC 20375
}

HOWARD A. SMITH

Laboratory for Astrophysics, National Air and Space Museum, Smithsonian Institution, Washington, DC 20560

MARCEL KLAPISCH

Artep Inc., Naval Research Laboratory, Code 4694, Washington, DC 20375

ANAND K. BHATIA

NASA/Goddard Space Flight Center, Greenbelt, MD 20771

and

AVI BAR-SHALOM

Nuclear Research Center of the Negev, P.O. Box 9001, Beer Sheva, Israel

Infrared coronal emission lines are providing a new window for observation and analysis of highly ionized gas in Galactic and extragalactic sources such as Seyfert nuclei and classical novae shells. These lines are expected to be primary coolants in colliding galaxies, galaxy cluster cooling flows, cometary-compact HII regions, and supernova remnants. In this poster, we summarize results discussed in detail by Greenhouse et al. 1993, ApJS, 88, 23. We discuss approximately 74 infrared $(1<\lambda \mu \mathrm{m}<280)$ transitions within the ground configurations $2 s^{2} 2 p^{k}$ and $3 s^{2} 3 p^{k}$ ( $k=1$ to 5 ) or the first excited configurations $2 s 2 p$ and $3 s 3 p$ of highly ionized $(\chi \geq 100 \mathrm{eV}) \mathrm{O}, \mathrm{Ne}, \mathrm{Na}, \mathrm{Mg}, \mathrm{Al}, \mathrm{Si}, \mathrm{S}, \mathrm{Ar}, \mathrm{Ca}, \mathrm{Fe}$, and $\mathrm{Ni}$. We present results from detailed balance calculations, critical densities for collisional de-excitation, intrinsic photon rates, branching ratios, and excitation temperatures for the transitions. The temperature and density parameter space for dominant cooling via infrared coronal lines is presented, and the relationship of infrared and optical coronal lines is discussed.

We find that under physical conditions found in Seyfert nuclei, 14 of 70 transitions examined have significant population inversions. Laser gain lengths and corresponding column densities are calculated. We find that several infrared coronal line transitions have laser gain lengths that correspond to column densities which are modeled to exist in Seyfert nuclei. Observations that can reveal inverted level populations and laser gain in infrared coronal lines are also suggested. 